\title{
New Mission Concept: Investigation of the Galactic Center with the Galactic Center Explorer (GalCenEx)
}

\author{
Alexander Moiseev ${ }^{1}$ \\ CRESST/NASA/GSFC and University of Maryland, College Park \\ UMCP, College Park, MD 20772, USA \\ E-mail: alexander.a.moiseevenasa.gov
}

Elizabeth Hays, David Thompson

NASA/Goddard Space Flight Center

8800 Greenbelt Rd, Greenbelt, MD 20771, USA

\section{Carolyn Kierans}

NPP/NASA/Goddard Space Flight Center

8800 Greenbelt Rd, Greenbelt, MD 20771, USA

The European Space Observatory INTEGRAL has been providing excellent results on X-ray and $\gamma$-ray astronomy since its launch in 2002. Among the most important results are the spectroscopy and imaging measurements of celestial $\gamma$-ray sources, including mapping the $511 \mathrm{keV}$ radiation from the Galactic plane. However, the nature of the $511 \mathrm{keV}$ positron annihilation line around the Galactic Center region is still a mystery. Similarly, the structure and nature of the Galactic Center supermassive black hole and its surroundings, as well as the structure of heavily populated sky regions such as Cygnus and Carina in $\gamma$-rays, remain unclear. We present a concept of a potential next-generation $\gamma$-ray telescope with the major science objectives: a) solve the mystery of the Galactic Center region $511 \mathrm{keV}$ positron annihilation line by mapping the Galactic Center region with high energy ( $<1 \%$ FWHM) and angular $\left(<10\right.$ arcmin) resolution and with line sensitivity $\left.<10^{-5} \mathrm{ph} \mathrm{cm}^{-2} \mathrm{~s}^{-1} ; \mathrm{b}\right)$ understand the nature of the Galactic Center supermassive black hole environment by creating a high-resolution spectral and intensity map of the surrounding region in $0.1-20 \mathrm{MeV} \gamma$ rays; and c) explore Galactic chemical evolution and sites of explosive element synthesis by conducting high-sensitivity measurements of nuclear lines from Type 1a supernovae and other objects. The instrument is based on a novel highenergy and position resolution CdZnTe Imager, coded aperture mask, and heavy-scintillator shield, and can be considered for a NASA Explorer mission.

36th International Cosmic Ray Conference -ICRC2019-

July 24th - August 1st, 2019

Madison, WI, U.S.A. 


\section{Introduction: Motivation and Science Objectives}

The mysterious nature of the Galactic Center (GC) has been puzzling scientists for decades. There have been a number of different missions to investigate the supermassive black hole at the center of our Galaxy and its vicinity, but several key questions are still unanswered. Among them are the unresolved nature of the $511 \mathrm{keV}$ positron annihilation line, the origin and nature of the $\gamma$ ray excess at the GC, and the nature of unassociated $\gamma$-ray sources ( $4^{\text {th }}$ Fermi Gamma-ray Catalog 4FGL has 1323 unassociated sources out of 5098 detected, with the majority of them in the Galactic plane near the GC [1]). In order to progress in our understanding of the GC in the $\mathrm{MeV}$ energy range, an instrument with angular resolution of the order of arcmins and energy resolution of the order of $1 \%$ is necessary. The only currently feasible way to achieve such angular resolution for $\mathrm{MeV}$ photons is to use the coded aperture method. This method has been successfully used in many $\mathrm{X}$ - and $\gamma$-ray experiments, see e.g. [2, 3, 4 and references therein], but requires a highperformance position-sensitive detector. The energy resolution of $1 \%$ or better can be provided by either Ge or CdZnTe detectors. Currently being developed for the AMEGO mission [5], a CdZnTe Imaging Calorimeter based on the novel Frisch-grid bar CZT detectors [6] uniquely meets these requirements, providing a fraction of $\mathrm{mm}$ position resolution along with $<1 \%$ energy resolution at $1 \mathrm{MeV}$.

Being strongly motivated by these challenging and exciting scientific perspectives, and the availability of a recently developed suitable detector, we are developing the Galactic Center Explorer (GalCenEx) space mission to pursue the following three main science objectives:

- Investigate the Galactic Center region $511 \mathrm{keV}$ positron annihilation line by mapping the Galactic Center with high energy $(<1 \%$ FWHM) and angular $(<10$ arcmin) resolution and with line sensitivity $<10^{-5} \mathrm{ph} \mathrm{cm}^{-2} \mathrm{~s}^{-1}$ with $10^{6} \mathrm{~s}$ observation;

- Understand the nature of the Galactic Center supermassive black hole environment and surrounding region by creating an intensity map with high spectral and spatial resolution for the photon energy $0.1-20 \mathrm{MeV}$;

- Investigate heavily populated sky regions such as Cygnus and Carina to reveal their fine spatial and spectral structure, with similar objectives as for the Galactic Center.

In addition to the science goals listed above, GalCenEx will be able to contribute to the field of $\mathrm{MeV}$ astrophysics with other observations:

- Explore Galactic chemical evolution and sites of explosive element synthesis by conducting high-sensitivity measurements of nuclear lines from Type 1a supernovae and from other objects;

- Provide measurement of $\gamma$-ray flux polarization for different classes of sources: blazars, pulsar systems, gamma-ray bursts;

- Probe the origin of the Fermi bubbles by extracting the diffuse $\mathrm{MeV}$ spectrum from the base of the bubbles, exploiting high angular resolution to separate the unresolved point source contamination. 


\section{Instrument: Design and Approach}

The GalCenEx concept is based on two driving requirements: achieve high energy $(<1 \%$ FWHM at $1 \mathrm{MeV})$ and angular ( $<10$ arcmin) resolution, and high line sensitivity $\left(<10^{-5} \mathrm{ph} \mathrm{cm}^{-2}\right.$ $\left.\mathrm{s}^{-1}\right)$. The energy resolution is determined by the detector system, and thus high-spectral resolution semiconductor detectors are a prime candidate. Excellent angular resolution can be achieved through the use of a coded aperture mask. The sensitivity depends on the detecting system but also on the level of residual background, and GalCenEx design will be optimized to minimize it.

\subsection{Energy Resolution}

Solid-state detectors, based on Ge or CdTe (CdZnTe) semiconductors, undoubtedly provide the best energy resolution for $\gamma$-rays in the $\mathrm{MeV}$ energy range. Ge-based detectors have the best parameters but require liquid nitrogen cooling. We select a CdZnTe-based detector for GalCenEx, which demonstrates slightly worse energy resolution than $\mathrm{Ge}$ (the best results for CdZnTe show $0.4 \%$ FWHM at $662 \mathrm{keV}$ [7]), but CdZnTe operates at ambient temperature. This feature makes it preferable for use in space instruments, eliminating the need for a cryogenic system.

\subsection{Angular Resolution}

Imaging in the $\mathrm{MeV}$ energy range $0.1-20 \mathrm{MeV}$ is complicated by competing processes of photon interaction: Compton scattering and pair production (above $1.022 \mathrm{MeV}$ ). While Compton telescopes take advantage of the dominant process at lower energies, their angular resolution is fundamentally limited by Doppler broadening. Therefore, it is extremely difficult to achieve better than $\sim 1$ degree angular resolution with direct photon detection.

Coded aperture mask (CAM) provides the only currently feasible way to provide an arcminlevel of angular resolution in the $\mathrm{MeV}$ range. The mask consists of an array of randomly positioned opaque and transparent elements, which is set in the field of view of the instrument, in front of the position sensitive detector. Each possible source position within the field of view creates a unique shadow on the position sensitive detector, and the direction to the source can be determined by a variety of methods based on cross-correlation of the mask image and mask element pattern $[2,8]$. The angular resolution of this technique is defined, in the first approximation, by the ratio of the mask element size to the distance between the mask and the detector plane. This can be made arbitrarily small, but the mask element should be no smaller than 2-3 times the intrinsic detector position resolution to achieve sufficient signal-to-noise ratio and consequently accurate source localization.

By combining a coded aperture mask with a Compton telescope detector plane, GalCenEx takes advantage of both detection methods and allows one to achieve excellent angular resolution while maintaining the background rejection capabilities. 


\subsection{Background Suppression}

Backgrounds in the $\mathrm{MeV}$ regime are notoriously high, and the effect of their various components can be very damaging to the sensitivity. Special attention in GalCenEx design will be paid to the "unremovable in analysis" photons from intrinsic activation of the instrument induced by charged cosmic rays and neutrons. Another source of MeV-background is a variety of spurious or misreconstructed events, but here we use a power of a background rejection through Compton event reconstruction: therefore, the GalCenEx design combines advantages of Compton telescopes and coded aperture masks.

To minimize the effect of background from activation and intrinsic radiation, we will carefully select the materials used in the instrument. We will minimize the usage of any metals, replacing them by the composites (thanks to rapidly developing technology) and non-metals, aiming to have only the unavoidable small amount of copper and silicon in electronics. In order to reduce the significant photonic background from Earth's atmosphere, as well as from diffuse Galactic radiation, the instrument fully-coded aperture will be shielded by an anti-coincidence scintillator, chosen here to be BGO. Furthermore, and very importantly, GalCenEx will fly in an equatorial Low-Earth Orbit to reduce instrument activation from the South Atlantic Anomaly [9].
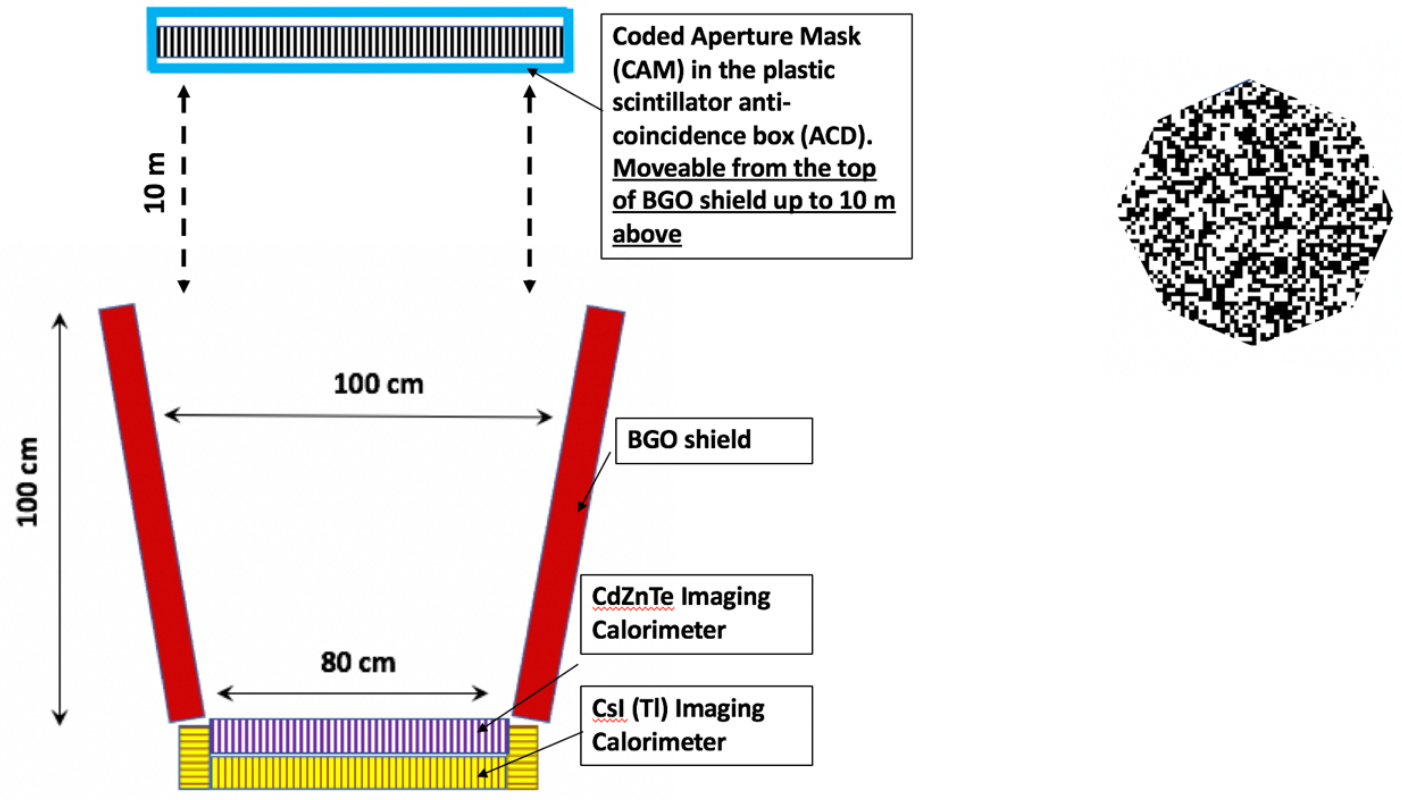

Figure 1. GalCenEx conceptual design (schematic view, left panel). It can be imagined as an octagonal box of heavy scintillator BGO, open at both ends and increasing in width from the detector plane toward the mask. The schematic top view of mask is shown on the right. The CdZnTe Imager will has the same octogonal cross-section built up with detector modules. 


\begin{tabular}{|c|c|}
\hline Detector Area & $10,000 \mathrm{~cm}^{2}$ \\
\hline Detector thickness & $\begin{array}{l}42 \mathrm{~g} / \mathrm{cm}^{2} \text { (consists of } 17 \mathrm{~g} / \mathrm{cm}^{2} \mathrm{CdZnTe} \text {, and } 25 \mathrm{~g} / \mathrm{cm}^{2} \mathrm{CsI} \text { ), or } \\
4.6 \mathrm{X}_{0} \text { (consists of } 1.9 \mathrm{X}_{0} \mathrm{CdZnTe} \text { and } 2.7 \mathrm{X}_{0} \mathrm{Csl} \text { ) }\end{array}$ \\
\hline \multirow[t]{2}{*}{ Effective Area (1 MeV) } & $\sim 2,500 \mathrm{~cm}^{2}$ (Mask mode) \\
\hline & $\sim 500 \mathrm{~cm}^{2}$ (Compton/combined mode) \\
\hline Energy range & $0.1-20 \mathrm{MeV}$ \\
\hline \multirow[t]{3}{*}{ Angular Resolution } & $10 \operatorname{arcmin}$ (mask in default position) \\
\hline & $1 \operatorname{arcmin}($ mask at $10 \mathrm{~m})$ \\
\hline & $5-10^{\circ}$ (Compton mode) \\
\hline Energy resolution (1 MeV) & $<1 \%$ FWHM \\
\hline Instrument mass & $\sim 2,000 \mathrm{~kg}$ \\
\hline
\end{tabular}

Table 1. GalCenEx instrument parameters and expected performance (simulated with MEGAlib [10]).

\section{Instrument: Conceptual Design and Subsystems}

A schematic diagram of the GalCenEx telescope concept is shown in Fig.1, and its expected performance is summarized in Table 1. GalCenEx consists of 5 sub-systems: 1) the CAM modulates the incident $\gamma$-ray flux and a shadow is created on the imaging calorimeter, 2) the CdZnTe imaging calorimeter provides a position of the first interaction of the arrived photons (to reconstruct the image of the mask), and measures its energy with high accuracy, 3) the CsI bar calorimeter surrounds CdZnTe calorimeter on the bottom and four sides contains high-energy events, 4) the detector plane is surrounded by an octagonal box of heavy scintillator BGO, open at both ends and increasing in width toward the mask, which provides active shielding from side-entering photons, and 5) the CAM is surrounded by an anti-coincidence detector (ACD) to reject charge particle backgrounds.

Different configurations of events detected by GalCenEx are schematically shown in Fig. 2. For lower energy events, a photon which passes through the CAM can photoabsorb in the CdZnTe calorimeter and can be treated as a standard coded-aperture mask event. This we refer to as CAM mode. For higher-energy photons, GalCenEx can operate in the Compton mode: a photon is detected as interacting more than once in the imaging calorimeter and reconstructed using the measured positions and energies. An example of a Compton mode event is shown in Fig. 2, where Event 1 requires the interactions 1 through 4 to be measured for proper reconstruction. The field of view for the combined Compton and CAM mode corresponds to the solid angle of the CAM. The ability to combine both the CAM and Compton operation modes provides the best source localization and resolution of closely spaced sources [11]. 


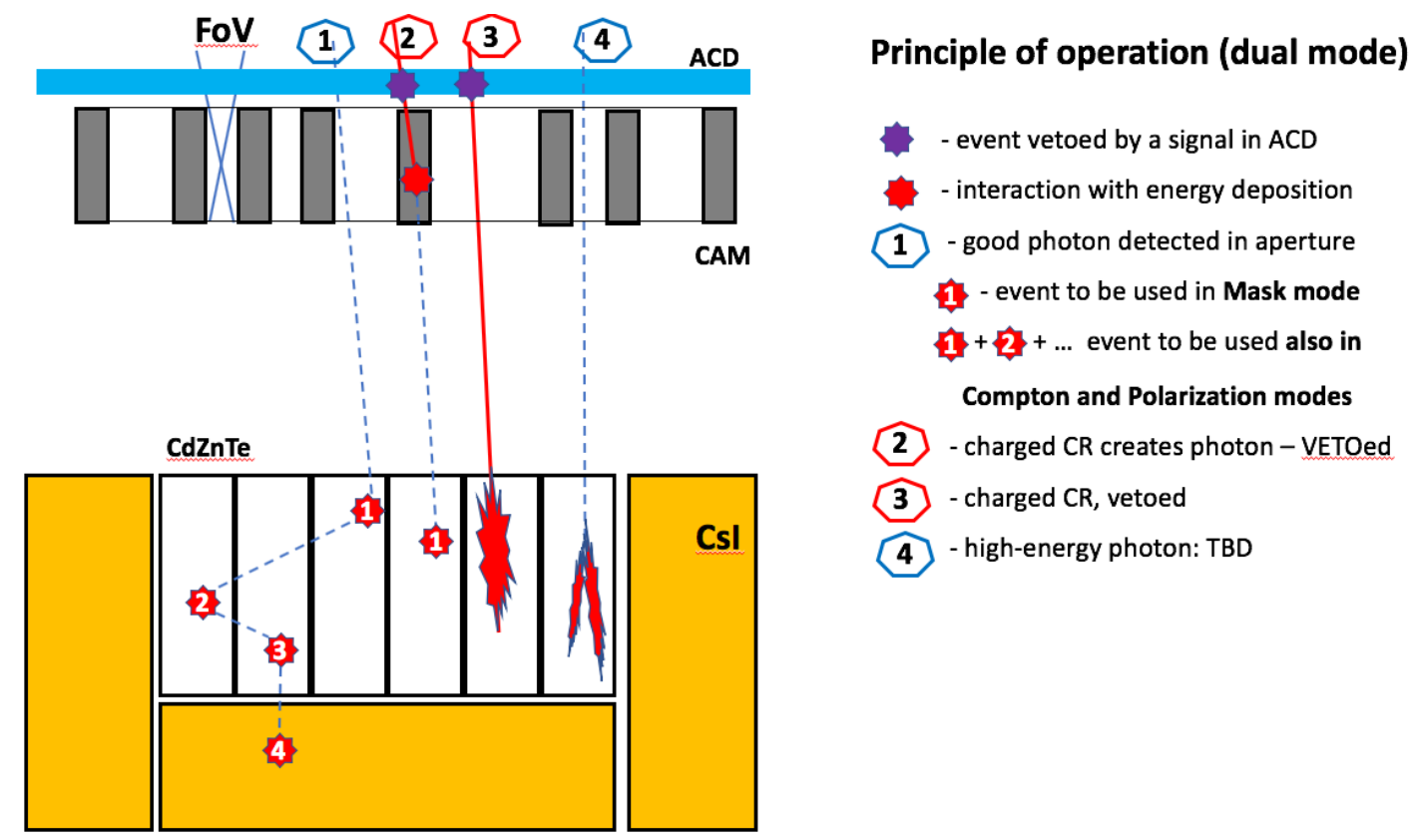

Figure 2. Event configurations for GalCenEx. Event types labeled by 1 and 4 are good events that can be measured by GalCenEx and reconstructed. Event types labeled by 2 and 3 show two ways that charged cosmic ray interactions can be vetoed by the ACD.

\subsection{CdZnTe Imaging Calorimeter}

The imager consists of an array of $8 \mathrm{~mm}$ x $8 \mathrm{~mm} \times 30 \mathrm{~mm}$ CdZnTe bars. The bars are combined into 4-by-4-bar modules served by an individual ASIC. The imager provides a precise measurement of the location and energy of the incident photon to create the image of the mask, and also enables the Compton mode of GalCenEx operation by measuring the position and energy of the scattered Compton photon, used for event reconstruction. The CdZnTe bars are operated in a drift mode that enables 3-dimensional reconstruction of the location of the interaction in the detector, thus providing excellent positional resolution of $<1 \mathrm{~mm}$, as well as good energy resolution $(<1 \%$ at $662 \mathrm{keV}$ ) at room temperature. In the event of several interactions in a single bar, the position is given by "center of gravity" of the multiple energy depositions, and although the total deposited energy is still measured, the Compton reconstruction of the event is much more complicated. We are investigating the use of a wave-front sampling ASIC [12] to enable the detection of multipleinteraction events. 


\subsection{Coded-Aperture Mask}

The mask pattern is currently based on the Uniformly Redundant Array (URA) algorithm (see e.g. [ 8]) but subject to be optimized later. The element size is chosen to be $3 \mathrm{~mm}$; the mask thickness is $15 \mathrm{~mm}$ of tungsten $\left(4 \mathrm{X}_{0}\right)$. The mask parameters have been chosen as a trade-off between instrument angular resolution, signal-to-noise ratio in the mask image reconstruction, and instrument field of view. In order to eliminate the photons induced by incident cosmic rays in the mask material (tungsten), the CAM is surrounded by a plastic scintillator ACD which generates a veto signal if a charged cosmic-ray particle hits the mask.

In the default position, the mask resides on top of the BGO shield, supported by the extendible mast. The extendible mast can bring the mask to any distance from the top of the BGO shield, up to $10 \mathrm{~m}$, providing better angular resolution. We are considering the option in which the mask will be able to rotate for further improvement of the instrument imaging capability (mask - no mask approach).

\subsection{BGO shield}

A BGO shield detector surrounds the imaging calorimeter and CAM, in its default location. The BGO detector consists of 8 trapezoidal panels, made of $4 \mathrm{~cm}$-thick BGO scintillator, read-out by silicon photomultipliers (SiPMs) distributed along the scintillator edges. The opening angle corresponds to the fully-coded aperture angle and is equal to the ratio of the mask element size to its height (mask thickness). The shield completely seals the instrument aperture (fully-coded field of view) from photons arriving outside of the aperture.

\subsection{CsI(TI) Calorimeter}

The concept for the CsI Calorimeter is similar to that of CdZnTe imager (but replacing expensive CdZnTe detectors by CsI), and also to the concept of CsI calorimeter of eASTROGAM [13]. It is made of $5 \mathrm{~mm} \times 5 \mathrm{~mm} \times 5 \mathrm{~cm}$ CsI(Tl) bars, individually read out from both ends by SiPMs. Although the energy ( $\sim 5 \%$ accuracy) and position $(\sim 1 \mathrm{~cm}$ accuracy) resolution of the CsI calorimeter are worse than the $\mathrm{CdZnTe}$, it increases the effective area at higher energies when combined with the CdZnTe calorimeter since it fully contains the tracks of high energy photons. It also retrieves photons which are not detected by the CdZnTe Imager.

\section{Summary}

Intriguing science puzzles in heavy populated sky regions, including our own Galactic Center, remain in the $\mathrm{MeV}$ range. With realistic perspectives in the successful development of a 3D position-sensitive high-energy resolution imager based on drift CdZnTe bar approach, we have developed the next-generation space $\gamma$-ray telescope GalCenEx. Combining the coded aperture mask technique with a Compton telescope detector plane may be the only viable approach to investigate the $\gamma$-ray sky with arcminute angular resolution, high sensitivity, and excellent energy resolution. Our concept inherits from the successful COMPTEL, Fermi-LAT, and INTEGRAL space missions and will be able to provide critical information in high-energy astrophysics 
with 1-2 orders of magnitude higher sensitivity than currently available. We are planning to propose GalCenEx in the next MIDEX NASA AO.

\section{References}

[1] https://fermi.gsfc.nasa.gov/ssc/data/access/lat/8yr catalog/

[2] E. Caroli, J.B. Stephen, G. Di Cocco, L. Natalucci, A. Spizzichino, Space Science Reviews 45 (1987) 349-403.

[3] P. Ubertini et al., IBIS: The Imager on-board INTEGRAL, A\&A 411, L131-L139 (2003)

[4] G. Vedrenne et al., SPI: The Spectrometer aboard INTEGRAL, A\&A 411, L63-70 (2003)

[5] A. Moiseev, All-sky Medium-Energy Gamma-ray Observatory (AMEGO), PoS(ICRC2019)583, These Proceedings

[6] A. Bolotnikov e al., Use of high-granularity position sensing to correct response non-unformities of CdZnTe detectors, APPLIED PHYSICS LETTERS 104, 263503 (2014)

[7] F. Zhang et al., 3-D Position Sensitive CdZnTe Spectrometer Performance Using Third Generation VAS/TAT Readout Electronics, IEEE Transactions on Nuclear Science, v. 52, 5, 2005

[8] G. Skinner, Imaging with coded-aperture masks, NiM in Physics Research 221 (1984), 3340

[9] P. Cumani et al., Background for a gamma-ray satellite on a low-Earth orbit, Experimental Astronomy https://doi.org/10.1007/s10686-019-09624-0, 2019

[10] A. Zoglauer, R. Andritschke, and F. Schopper. MEGAlib The Medium Energy Gamma-ray Astronomy Library. New Astronomy Reviews, 50:629-632, October 2006.

[11] M. Galloway et al., arXiv: 1705.02652, 2017

[12] Y. Zhu and Z. He, 2012 NSS/MIC Proceedings, R04-3

[13] V. Tatishev et al, arXiv: 1608.03739, 2016 